Fetal Diagnosis and Therapy

\title{
Diagnostic Features of Myelomeningocele: The Role of Ultrafast Fetal MRI
}

\author{
David M. Mirsky ${ }^{\text {a }}$ Erin S. Schwartz ${ }^{\text {b }}$ Deborah M. Zarnow ${ }^{\text {b }}$ \\ ${ }^{a}$ Children's Hospital Colorado, University of Colorado School of Medicine, Denver, Colo., and \\ ${ }^{b}$ Children's Hospital of Philadelphia, Perelman School of Medicine at the University of Pennsylvania, \\ Philadelphia, Pa., USA
}

\section{Key Words}

Fetal magnetic resonance imaging - Myelomeningocele .

Chiari II malformation

\begin{abstract}
Fetal magnetic resonance imaging (MRI) is a useful adjunct to ultrasound in the evaluation of neural tube defects. The development of ultrafast imaging techniques has contributed to the increasing clinical use of fetal MRI. This review summarizes the utility, safety and technical aspects of fetal MRI and the role it plays in the evaluation of myelomeningocele. Emphasis is placed on the benefit of fetal MRI as it pertains to presurgical planning and perinatal management.
\end{abstract}

(c) 2014 S. Karger AG, Basel

\section{Introduction}

Imaging of neural tube defects has been transformed over the past 15 years with the utilization of fetal magnetic resonance imaging (MRI). While ultrasonography remains the primary imaging modality for the evaluation of neural tube defects, it has known limitations that may hamper accurate diagnoses such as in the setting of ma- ternal obesity, oligohydramnios and suboptimal fetal positioning. MRI is less significantly affected by these issues and can be useful to confirm suspected ultrasound findings and to exclude additional central nervous system abnormalities [1,2]. The development of ultrafast imaging techniques has contributed to the increasing clinical use of fetal MRI [3].

\section{Safety of Fetal MRI}

Fetal MRI is routinely performed on 1.5-TESLA scanners and, to date, there have been no proven harmful effects to the developing human fetus from limited exposure to the electromagnetic fields occurring during MRI $[4,5]$. The American College of Radiology does not stipulate any special consideration regarding MRI of the fetus at any stage of pregnancy [6]. In the USA, however, fetal MRI is generally not performed in the first trimester of gestation, given the theoretical concern for teratogenesis. Furthermore, it is difficult to acquire high-quality images in very young fetuses by MRI due to their small size and the fact that the major components of their central nervous system (such as the corpus callosum and cerebellar vermis) continue to develop well into the second trimester. In cen-

\section{KARGER 125}

(c) 2014 S. Karger AG, Base

$1015-3837 / 14 / 0373-0219 \$ 39.50 / 0$
Deborah M. Zarnow, MD

Division of Neuroradiology, Department of Radiology Children's Hospital of Philadelphia, Wood Room 2115 324 South 34th Street, Philadelphia, PA 19104 (USA)

E-Mail zarnow@email.chop.edu 
Fig. 1. a Sagittal T2 HASTE image of a fetus (19 weeks' gestation) demonstrates typical stigmata of Chiari II malformation with marked reduction of supratentorial CSF spaces (black arrow). There is severe crowding of the posterior fossa with effacement of the 4th ventricle and retrocerebellar CSF cistern and hindbrain herniation (white arrow). An open defect is seen in the lower lumbar spine with absence of skin and posterior elements and a posterior MMC sac with neural placode (bold white arrow). b Axial T2 HASTE image of the lower spine in the same fetus shows the eversion of the lamina, absence of the posterior elements and the presence of the neural placode beyond the plane of the fetal back consistent with an MMC (black arrow).
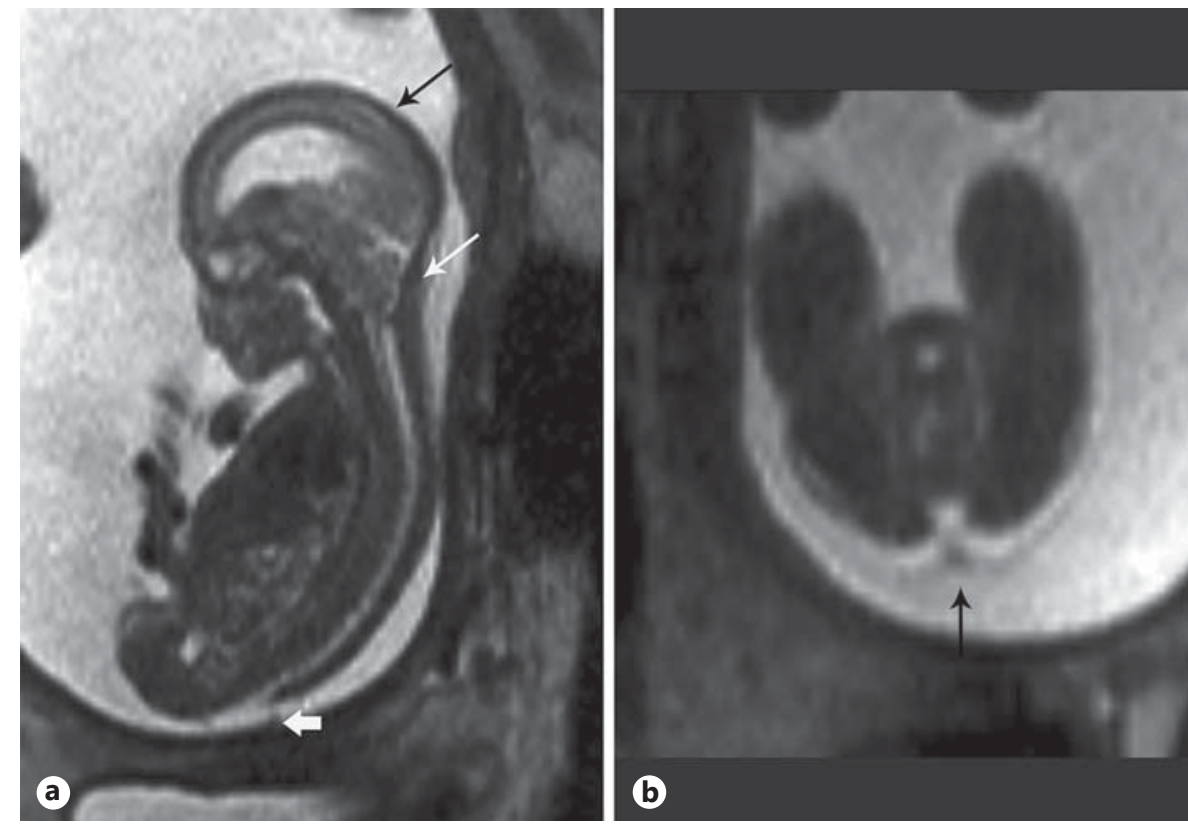

ters that perform in utero myelomeningocele (MMC) repair, most imaging occurs between 19 and 24 weeks of gestational age to allow for fetal surgery prior to 26 weeks' gestation [7].

\section{Technical Aspects}

Various types of coils can be used for fetal MRI. A body coil alone or a body phased-array coil in combination with a surface coil situated on the mother's abdomen is commonly used. Images are acquired in the sagittal, axial and coronal planes relative to the fetal spine. Fast imaging techniques allow for images to be obtained in a fraction of a second [3]. At the authors' institution, the half-Fourier single-shot turbo spin-echo (HASTE) sequence serves as the mainstay of the examination. HASTE sequences provide heavily $\mathrm{T} 2$-weighted images with low susceptibility weighting in very short acquisition times, enabling good discrimination of fetal central nervous system features. Sequential slice capability and interleaving allows for high-quality imaging despite fetal movement. Sequences with more sensitivity to focal paramagnetic susceptibility effects, such as extremely rapid gradient echo, echo planar imaging, can be very useful in the detection of hemorrhage and mineralization, which is important in the evaluation of the fetal brain in the setting of a Chiari II malformation. T1-weighted images have limited utility in the evaluation of fetal spinal dysraphism and brain morphol- ogy. Cine imaging, while not particularly useful in primary diagnosis, does provide prognostic information complementary to the ultrasound examination in assessing lower extremity movement, due to the concern for neurogenic dysfunction secondary to the MMC.

A typical fetal MRI examination takes approximately $40 \mathrm{~min}$. At the authors' institution, no sedation is provided to the mother or to the fetus. As fetuses with open neural tube defects have associated Chiari II malformations, the entire fetal central nervous system should be scanned. Absence of Chiari II stigmata should raise suspicion for a closed spinal dysraphism such as a lipomyelomeningocele or terminal myelocystocele.

Following a standard localizer sequence to determine fetal positioning, images are acquired in 3 orthogonal planes with respect to the fetal brain and spine. Each subsequent series is calibrated from the previous acquisition to attempt to account for interval fetal motion. Similar to postnatal imaging, the sagittal and coronal views often provide a good overview of the spinal dysraphism, while the axial images allow for detailed analysis of the placode and posterior osseous defect. It should be noted that the normal fetal spine has a thoracic kyphosis. Compaction of the fetus in the maternal uterus often accentuates this curvature, making it difficult to image the spine in a single axial plane. To account for this, axial imaging is often performed in multiple separate stations, each of which is obtained perpendicular to the long axis of the spine. 
Fig. 2. a Sagittal T2 HASTE image of a fetus (20 weeks and 3 days' gestation) demonstrates typical stigmata of Chiari II malformation with marked reduction of supratentorial CSF spaces (black arrow). There is severe crowding of the posterior fossa with effacement of the 4th ventricle and retrocerebellar CSF cistern and hindbrain herniation (white arrow). An open defect is seen in the upper lumbar spine with absence of skin and posterior elements with the low-lying spinal cord and the neural placode extending below the level of the defect (bold white arrow). b Axial T2 HASTE image of the lumbar spine in the same fetus shows eversion of the lamina, absence of the posterior elements and sac and the presence of the neural placode within the plane of the fetal back consistent with a myeloschisis (black arrow).
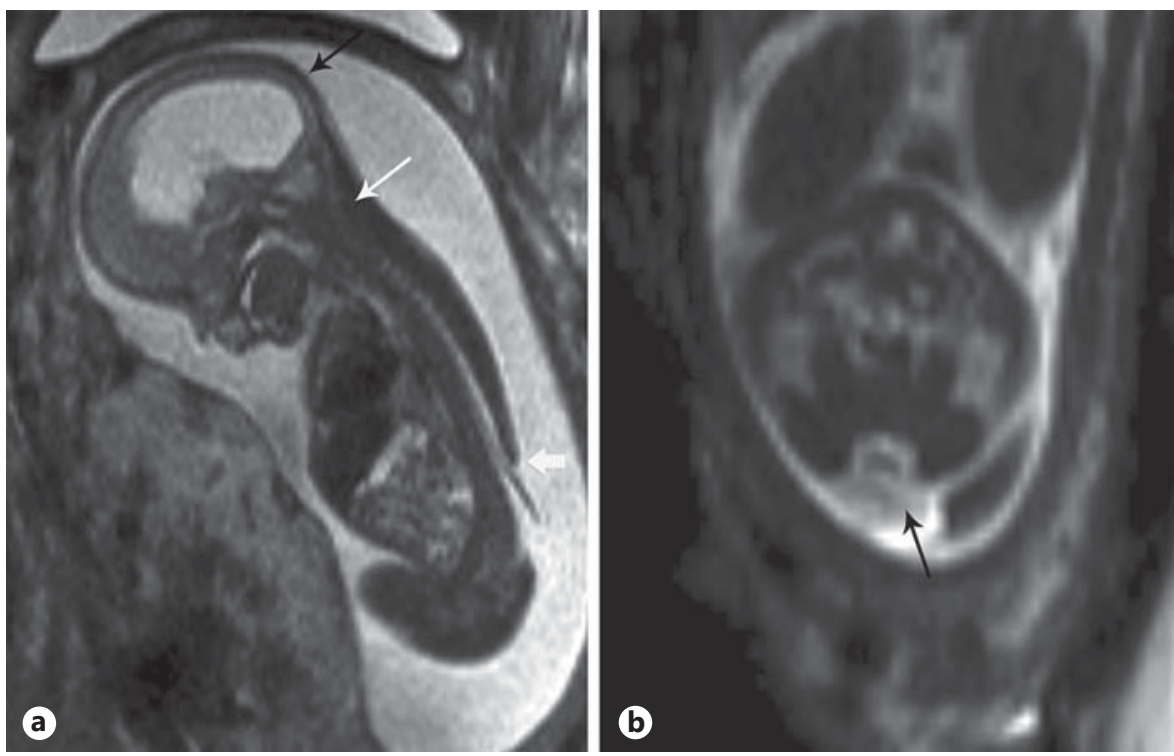

\section{Open Spinal Dysraphism}

The appearance of the MMC on fetal MRI reflects the embryological disturbance of primary neurulation [8]. There is failure of normal neuroectodermally derived neural tube dysjunction from the adjacent cutaneous ectoderm during the period of expected neural tube closure, resulting in a flat midline neural placode. It is the exposed, posterior placode surface that reflects the internal neural tube ependyma.

The majority of MMCs occur in the lumbar region, though they can occur anywhere along the spine. When the spinal cord continues distally, beyond the level of the dysraphism, the neural placode is considered segmental, and when the placode occurs at the end of the spinal cord it is considered terminal.

In the axial plane, the open dysraphism is seen as everted laminae with deficiency of the overlying muscle and skin. The neural placode is typically elevated beyond the confines of the dysraphic defect due to expansion of the underlying subarachnoid CSF space, constituting an MMC proper (fig. 1). When there is no subarachnoid space expansion, the placode remains flush with the defect or within the confines of the spinal canal and is classified as a myeloschisis, also known as myelocele (fig. 2) [8]. While both MMC and myeloschisis reflect a similar underlying embryological disturbance, accurate delineation between the two is important for fetal surgical planning.

Diagnostic Features of MMC: The Role of Ultrafast Fetal MRI

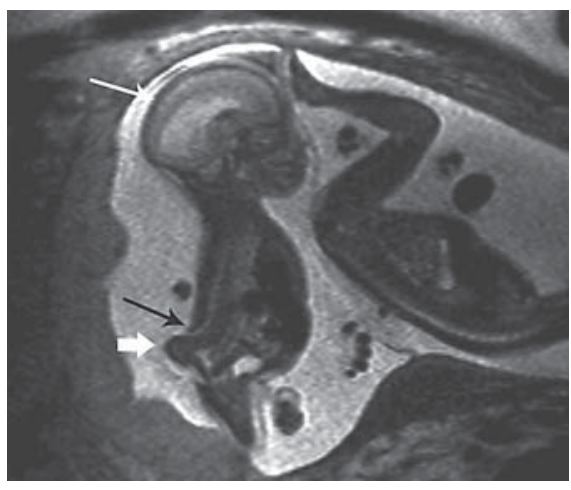

Fig. 3. Sagittal T2 HASTE image of a twin fetus (21 weeks' gestation) demonstrates typical stigmata of Chiari II malformation with marked reduction of supratentorial CSF spaces (white arrow). An open defect is seen in the lower lumbar spine with absence of skin and posterior elements (black arrow). Just below the defect there is a marked focal kyphosis of the lumbar spine (bold white arrow). The co-twin did not have an open spinal dysraphism.

In assessing an open spinal dysraphism, it is not only important to define the defect but to also look for associated abnormalities. Kyphoscoliosis may be present due to neuromuscular imbalance with or without vertebral segmentation anomalies, the latter of which includes hemivertebrae or a bony bar (fig. 3) [9]. A split cord malformation may occur above, below or at the level of the MMC. Syrinx formation has been described in $30-75 \%$ of cases (fig. 4). 
Fig. 4. Coronal (a) and axial (b) T2 HASTE MMC (not shown) demonstrate abnormal high T2 signal centrally within the spinal cord consistent with syringohydromyelia (white arrows).

Fig. 5. Sagittal T2 HASTE image of a fetus strates typical stigmata of Chiari II malformation with marked reduction of supratentorial CSF spaces, severe crowding of the posterior fossa with effacement of the 4 th ventricle and retrocerebellar CSF cistern and hindbrain herniation. In addition, there is undulation of the margin of the lateral ventricles (white arrows) with white matter volume loss suggestive of prior injury in this fetus that also has severe congenital heart disease.

Fig. 6. a Axial T2 HASTE images of a fetus (23 weeks' gestation) with a Chiari II and MMC demonstrate multiple small focal hypointense nodules along the walls of the posterior horns of the lateral ventricles (white arrows) in keeping with subependymal gray matter heterotopia. b Axial T2weighted images of the same patient at 6 days of age confirm the presence of multiple nodules of subependymal gray matter heterotopia (white arrows). images of a fetus (23 weeks' gestation) with (25 weeks and 3 days' gestation) demon-
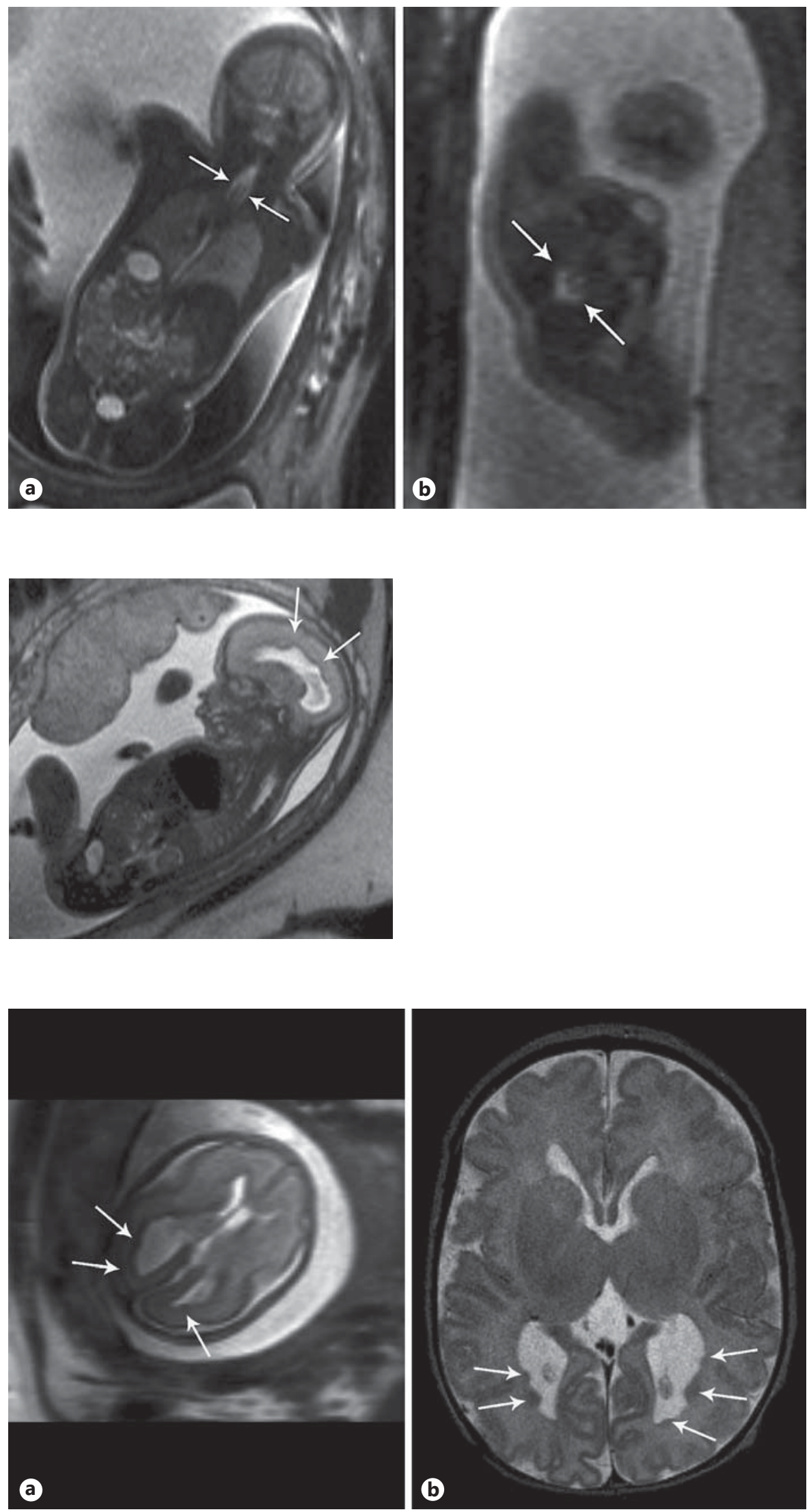


\section{Chiari II Malformation}

Imaging of the fetal brain is warranted in all instances of prenatal MMC diagnosis. The overwhelming majority of cases, but not all, are associated with the classic stigmata of Chiari II malformation such as a small, crowded posterior fossa, hindbrain herniation and tectal beaking [10]. While ultrasound is very good at delineating the major features of the malformation, MRI has been shown to provide better characterization of brain anomalies and superimposed, acquired insults (fig. 5). In the context of a Chiari II malformation, high-quality MRI is far supe- rior at detecting the subtle findings of subependymal gray matter heterotopia (fig. 6), small amounts of intraventricular blood products (fig. 7) and callosal dysgenesis [2, $3,11,12]$. Varying degrees of hindbrain herniation, a point of particular importance in centers performing fetal repair of MMC, can also be more accurately assessed with MRI. In our experience, MRI is more accurate than ultrasound in determining the presence or absence of hindbrain herniation, especially when residual CSF is seen within the retrocerebellar cistern (fig. 8). Consequently, MRI confirmation of hindbrain herniation is a prerequisite for fetal MMC repair candidacy.
Fig. 7. Axial echo planar images of a fetus (22 weeks and 5 days' gestation) with Chiari II and MMC with layering hypointensity in the lateral ventricles consistent with intraventricular hemorrhage (white arrows).

Fig. 8. a Sagittal T2 HASTE image of a fetus (20 weeks' gestation) does not demonstrate typical stigmata of Chiari II malformation. The supratentorial CSF spaces are not significantly reduced. Evaluation of the posterior fossa demonstrates slight crowding with preserved CSF space in the retrocerebellar CSF cistern and absence of hindbrain herniation (white arrow). b Axial T2 HASTE image of the lower spine in the same fetus shows eversion of the lamina, absence of the posterior elements and the presence of the neural placode beyond the plane of the fetal back consistent with a MMC (black arrow). There is slight thickening of the margins of the sac, suggesting that this sac may be partially skin covered.
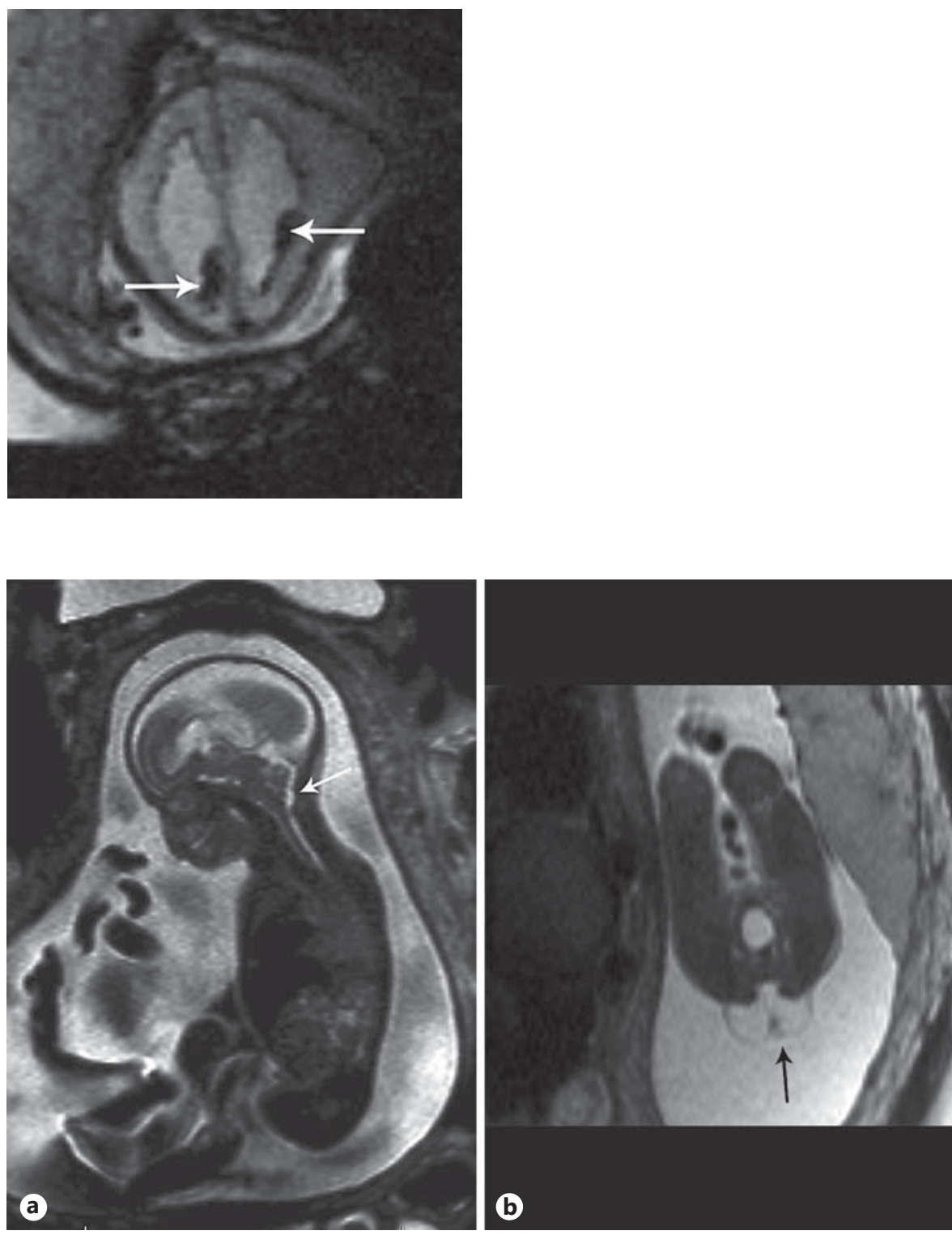
Fig. 9. a Sagittal T2 HASTE image of a fetus (23 weeks and 4 days' gestation) demonstrates typical stigmata of Chiari II malformation with marked reduction of supratentorial CSF spaces (black arrow). There is severe crowding of the posterior fossa with effacement of the 4 th ventricle and retrocerebellar CSF cistern and hindbrain herniation (white arrow). b Sagittal T2 HASTE image of the spine of the same fetus demonstrates an open defect in the lower lumbar spine with absence of skin and posterior elements with the low-lying spinal cord and the neural placode extending below the level of the defect within the plane of the fetal back consistent with a myeloschisis (white arrow). c Follow-up MR examination at 28 weeks of the same fetus following in utero MMC repair 4 weeks previously demonstrates improved visualization of the supratentorial CSF spaces (black arrow). The CSF spaces in the posterior fossa are now visualized, including the 4 th ventricle and retrocerebellar CSF cistern. Hindbrain herniation has resolved (white arrow). d Sagittal T2 HASTE image of the spine again demonstrates resolution of hindbrain herniation (white arrow). While the spinal cord termination remains low, the plane of the back is now closed (bold white arrow).
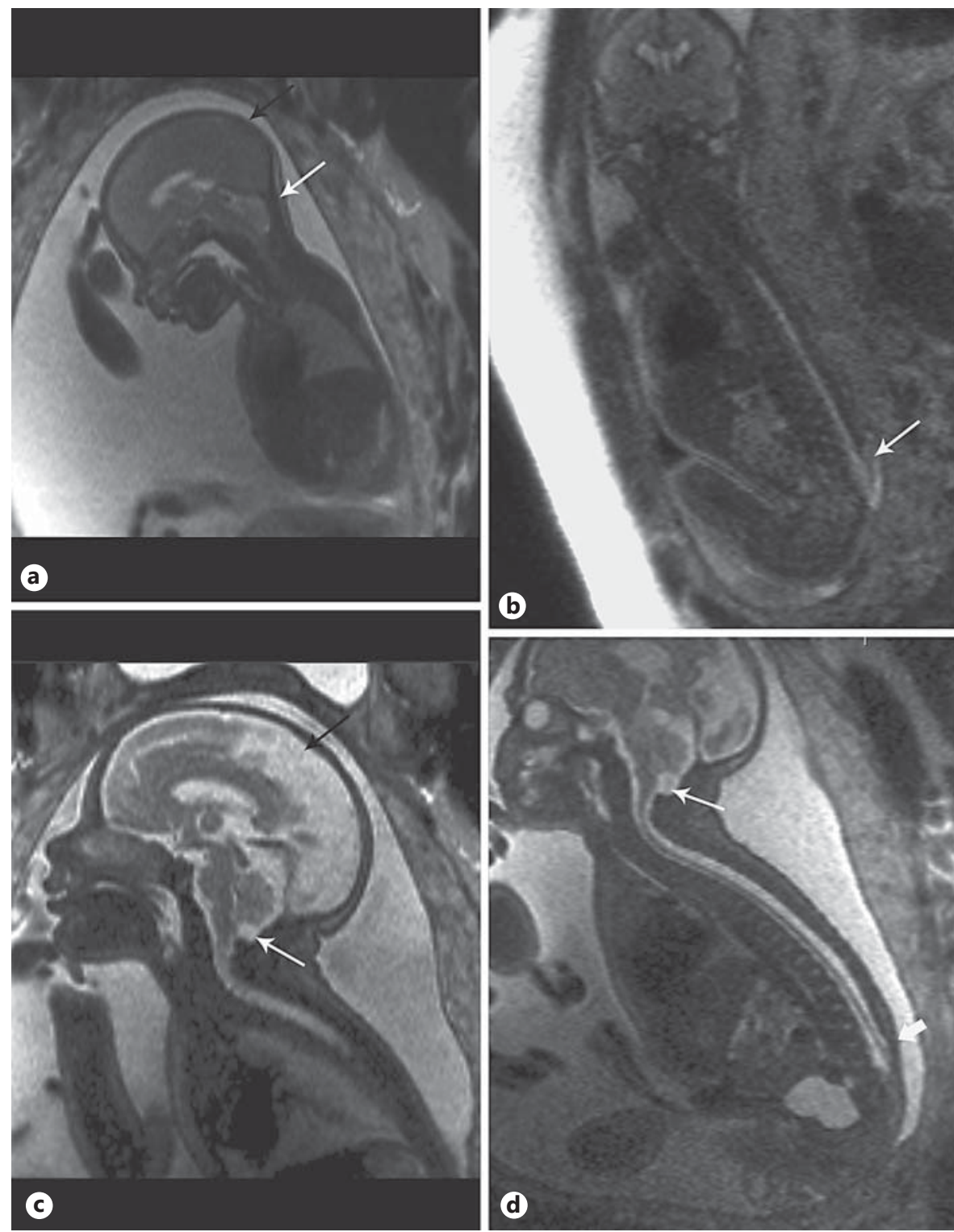

\section{Fetal Surgery}

With the advent of fetal MMC repair, fetal MRI not only plays an important role in presurgical planning but also serves as a sensitive means of following surgical outcomes. Results from the first phase of the Management of Myelomeningocele Study (MOMS) showed a reduction in Chiari II features in those fetuses undergoing prenatal repair versus those undergoing postnatal repair, most importantly in the degree of hindbrain herniation (fig. 9) [7]. Postrepair fetal imaging can serve as a baseline for comparison with postnatal imaging [13]. It is important to note that the spinal cord will remain permanently low- lying, despite prenatal or postnatal repair, but that a lowlying spinal cord termination on MRI does not always equate to clinical spinal cord retethering.

\section{Summary}

High-quality ultrafast fetal MRI has become a standard tool in the armamentarium of radiologists in evaluating MMCs, providing a valuable supplement to sonography and helping to guide diagnosis and management. Advances in fetal medicine and surgery have further solidified the role of MRI in the field of prenatal imaging. 


\section{References}

1 Coakley FV, Glenn OA, Qayyum A, Barkovich AJ, Goldstein R, Filly RA: Fetal MRI: A developing technique for the developing patient. AJR Am J Roentgenol 2004;182:243252.

$>2$ Levine D, Barnes PD, Madsen JR, Li W, Edelman RR: Fetal central nervous system anomalies: MR imaging augments sonographic diagnosis. Radiology 1997;204:635-642.

3 Simon EM, Goldstein RB, Coakley FV, Filly RA, Broderick KC, Musci TJ, Barkovich AJ: Fast MR imaging of fetal CNS anomalies in utero. AJNR Am J Neuroradiol 2000;21: 1688-1698.

$\checkmark 4$ Schwartz JL, Crooks LE: NMR imaging produces no observable mutations or cytotoxicity in mammalian cells. AJR Am J Roentgenol 1982;139:583-585.

$\checkmark 5$ Thomas A, Morris PG: The effects of NMR exposure on living organisms. I. A microbial assay. Br J Radiol 1981;54:615-621.
6 American College of Radiology (ACR); Society for Pediatric Radiology (SPR): ACR-SPR practice guideline for the safe and optimal performance of fetal magnetic resonance imaging (MRI). 2010, 2014.

7 Adzick NS, Thom EA, Spong CY, Brock JW, Burrows PK, Johnson MP, Howell LJ, Farrell JA, Dabrowiak ME, Sutton LN, Gupta N, Tulipan NB, D'Alton ME, Farmer DL; MOMS Investigators: A randomized trial of prenatal versus postnatal repair of myelomeningocele. N Engl J Med 2011;364:993-1004.

8 Tortori-Donati P, Rossi A, Cama A: Spinal dysraphism: a review of neuroradiological features with embryological correlations and proposal for a new classification. Neuroradiology 2000;42:471-491.

$>9$ Sarwark JF: Kyphosis deformity in myelomeningocele. Orthop Clin North Am 1999;30: 451-455, viii-ix.
10 McLone DG, Knepper PA: The cause of Chiari II malformation: a unified theory. Pediatr Neurosci 1989;15:1-12.

11 Levine D, Barnes PD, Madsen JR, Abbott J, Mehta T, Edelman RR: Central nervous system abnormalities assessed with prenatal magnetic resonance imaging. Obstet Gynecol 1999;94:1011-1019.

12 Saleem SN, Said AH, Abdel-Raouf M, El-Kattan EA, Zaki MS, Madkour N, Shokry M: Fetal MRI in the evaluation of fetuses referred for sonographically suspected neural tube defects (NTDS): impact on diagnosis and management decision. Neuroradiology 2009;51:761772 .

13 Sutton LN, Adzick NS, Bilaniuk LT, Johnson MP, Crombleholme TM, Flake AW: Improvement in hindbrain herniation demonstrated by serial fetal magnetic resonance imaging following fetal surgery for myelomeningocele. JAMA 1999;282:1826-1831. 